\title{
Twenty Years of Hepatitis $C$ in the Treviso District (Local Health Unit 2): Treatments, Clinical Management and Cost Analysis
}

Global \& Regional Health Technology

Assessment

Volume 2019: 1-9

(C) The Author(s) 2019

Article reuse guidelines:

sagepub.com/journals-permissions

DOI: 10.1 | 77/22842403। 9835865

journals.sagepub.com/home/grh

(3)SAGE

\author{
Annachiara Bellin'(i), Giulia Franchin ${ }^{2}$, Jenny Bolcato ${ }^{2}$, Alessandra \\ Bettiol', Roberta Pirolo², Alberto Schiavon', \\ Pietro Giusti', Michele Tessarin ${ }^{3}$ and Alessandro Chinellato ${ }^{2}$
}

\begin{abstract}
Chronic hepatitis C virus ( $\mathrm{HCV}$ ) infection is a global health problem, and about $10-30 \%$ of patients develop cirrhosis or hepatocellular carcinoma several years after being infected. In past decades, treatment of HCV infection was based on peginterferon and ribavirin, which lead to a sustained virologic response (SVR) in only 50-60\% of patients. Since 2014 , direct acting antiviral (DAA) agents have been available. Patients administered DAA agents usually reach SVR in 12 weeks. The aim of this study was to estimate the cost analysis of these innovative drugs while also taking into account the total health expenditure for managing HCV infection. The pharmaceutical and hospitalisation databases of the Local Health Unit (ULSS2) of Treviso were retrospectively analysed between 1997 and 2016 for each HCV patient. During this twenty-year period, people affected by HCV totalled 2,949; 277 of these patients were treated with DAA and, of these, only $2 \%$ did not reach SVR. The HCV genotype Ib was the most common, accounting for $58 \%$ of the total patients. The treatment for HCV genotype 3 was associated with higher costs. The expenses for the new treatments were found to be significantly higher compared to those for the old ones (i.e., peginterferon and ribavirin). The average costs for a cycle of therapy were $€ 8,000$ and $€ 24,000$ for interferon and DAA therapy, respectively. Total health care costs associated with $\mathrm{HCV}$ (excluding DAA treatments) for an individual HCV infection patient were estimated to be $€ 32,000$. Our results confirm the high efficacy of DAA therapy. Furthermore, these agents improve the clinical conditions and reduce both the treatment cost and health care in patients with $\mathrm{HCV}$ infection.
\end{abstract}

\section{Keywords}

Hepatitis C, antiviral agents, fibrosis, Interferon Type I, health care costs

Date received: 7 June 2018; revised: 7 January 2019; accepted: 12 February 2019

\section{Introduzione}

Con una diffusione che coinvolge a livello mondiale circa 110 milioni di persone positive ad anticorpi anti-HCV (indicativi di una infezione passata o in corso) a cui si aggiungono circa 80 milioni di persone con HCV RNA (indicativo di una infezione in corso o cronica), la prevalenza globale di epatite $\mathrm{C}$ cronica (HCV) si attesta al $1,1 \%{ }^{1}$ In Italia l'incidenza dell'infezione da $\mathrm{HCV}$ ha avuto un andamento decrescente fino al 2008, per poi stabilizzarsi ad un tasso che oscilla tra lo 0,2-0,3 per 100,000 abitanti. ${ }^{2}$ Studi epidemiologici condotti negli anni '90 hanno definito 1'Italia come il Paese d'Europa occidentale con la più alta prevalenza dell'infezione da $\mathrm{HCV}^{3,4}$ Tale prevalenza si è mantenuta anche negli ultimi

'Dipartimento di Scienze del Farmaco, Università degli Studi di Padova, Padova, Italy

2U.O.C. Politiche del Farmaco e Governo della Spesa Farmaceutica, Azienda ULSS2, Distretto di Treviso, Treviso, Italy

${ }^{3}$ U.O.C Direzione Sanitaria, Azienda ULSS2, Distretto di Treviso,

Treviso, Italy

Corresponding author:

Bellin Annachiara, Dipartimento di Scienze del Farmaco, Università degli Studi di Padova, Via Don Tosatto 147, Mestre (Venezia), Italy.

Email: annachiara.bellin@aulss3.veneto.it 
anni,, 5 in particolare per i soggetti di età superiore a 60 anni.

L'evoluzione della patologia è molto lenta; i segni clinici si manifestano nelle fasi più avanzate dell'infezione cronica con fibrosi, cirrosi ed epatocarcinoma. Si può stimare una mortalità nel mondo di 350.000 persone/anno; oltre a ciò va aggiunta l'insorgenza di un maggior rischio di complicazioni extra epatiche causate dal virus stesso dell'epatite C. ${ }^{7}$

Prima del 2013 le terapie antivirali esistenti rallentavano il decorso della malattia, e di fatto non portavano a guarigione del paziente. ${ }^{8-11}$ Lo scenario che si è aperto dall'entrata in commercio dei primi antivirali ad azione diretta (DAA), ha visto una escalation di nuove molecole, sempre più specifiche ed efficaci, in grado di curare ciascun genotipo. Questa indubbia innovazione ha visto costi elevati sul fronte della spesa farmaceutica. ${ }^{12-14}$ Per esempio, in Veneto nel 2016, questi farmaci hanno rappresentato la seconda voce di spesa farmaceutica dopo le terapie oncologiche. ${ }^{12}$

Questi nuovi DAA sono sottoposti a modelli di rimborso negoziato denominati MEAs (Managed Entry Agreement): si tratta di accordi negoziali di condivisione del rischio stipulati tra l'Agenzia Italiana del Farmaco (AIFA) e la singola Azienda Farmaceutica attraverso un accesso condizionato al mercato dei nuovi farmaci.

Ad oggi, nell'ambito dei possibili MEAs applicabili, per i farmaci antiepatite $\mathrm{C}$ gli accordi adottati sono stati di tipo finanziario. Più precisamente si è applicato il capping, il quale prevede un rimborso massimo per trattamento, con la spesa eccedente pagata dall'impresa, o l'accordo "prezzo/volume", che prevedeva all'aumentare dei volumi una riduzione dei prezzi di cessione al SSN. Le soglie e la relativa riduzione percentuale del prezzo sono state stabilite negli accordi negoziali con AIFA.

Gli importi di pay-back che venivano versati alle Regioni e, successivamente, alle Aziende ospedaliere erano dati riservati che impedivano di conoscere il costo effettivo del farmaco. Attualmente tali farmaci sono stati inseriti nel fondo innovativi.

Obiettivo principale dello studio è stato quello di analizzare i costi assistenziali dell'epatite $C$, non limitandosi al breve periodo di utilizzo dei nuovi farmaci, ma estendendo l'osservazione anche a tutta la storia clinica del paziente, considerando anche i costi diretti dedicati all'assistenza dei pazienti affetti da epatite $\mathrm{C}$. In seconda battuta si è misurata l'efficacia dei diversi regimi di trattamento.

\section{Metodi}

\section{Disegno dello studio}

Lo studio è di tipo osservazionale retrospettivo. La popolazione arruolata è rappresentata da maschi e femmine di età uguale o maggiore ai 18 anni, residenti nel territorio di competenza dell'Az. ULSS 2 di Treviso affetti da HCV prevalenti tra il 1997 e il 2016, utilizzando come data index la prima data di una prestazione tracciante, ovvero ricoveri ospedalieri e/o prescrizioni farmaceutiche correlate al virus dell'HCV. Nel caso di farmaci aspecifici è stata associata anche la ricerca della carica virale nella banca dati degli esami ematochimici. Come criterio di inclusione nello studio, sono stati adottati i parametri di prescrizione farmaceutica e di ricovero ospedaliero indicati nella Tabella 1.

Infine, si sono ricercate anche le esenzioni per epatite virale, ma non sono state determinanti per la definizione della coorte in quanto non è specifica per l'infezione da $\mathrm{HCV}$.

L'efficacia della terapia è stata valutata tramite il raggiungimento della risposta virologica sostenuta (SVR), ovvero la negatività dell'HCV-RNA a 12 settimane dalla conclusione del trattamento. Infatti, per valutare l'efficacia del farmaco è necessario attendere le 12 settimane, in quanto questo è il tempo necessario in cui il virus diminuisce la propria carica virale del $99 \%$ rispetto al valore basale. ${ }^{15}$

È stato possibile stratificare questo outcome in base al genotipo, solo per le terapie del biennio 2015-2016, in quanto la scelta del farmaco era vincolata dal genotipo del paziente, mentre, per i regimi che prevedevano l'interferone, la ricerca del genotipo non era analogamente obbligatoria.

\section{Fonti database}

Lo studio si avvale di database amministrativi opportunamente anonimizzati, nel periodo compreso tra il 1997 e il 2016.

Per quanto riguarda la ricerca delle ospedalizzazioni, si sono utilizzati database contenenti le schede di dimissione ospedaliera (SDO) e quelli contenenti gli accessi al pronto soccorso (APS). Il criterio di selezione è stata una diagnosi correlata all'infezione di HCV (Tabella 1).

L'altra fonte di ricerca della popolazione è stato il database contenente le prescrizioni farmaceutiche, indipendentemente dal canale di dispensazione, in quanto i farmaci utilizzati per il trattamento dell'infezione da HCV (specifici e non specifici) possono essere distribuiti direttamente (DD), per conto (DPC) e in regime convenzionale. Il criterio con cui sono stati selezionati i pazienti era la ricerca delle prescrizioni farmaceutiche per mezzo del codice ATC (Tabella 1). Si vuole specificare che la ricerca di farmaci come l'interferone e la ribavirina non erano prestazioni traccianti e univoche come tali per il trattamento dell'epatite $\mathrm{C}$, dato che sono utilizzati anche per altre patologie non di carattere virale, quindi per questi pazienti sono state ricercate altre condizioni concomitanti che permettessero l'entrata nello studio, come per esempio ospedalizzazione per epatite o ricerca della carica virale. La condizione determinante è stata 
Tabella I. Codici utilizzati per l'identificazione della popolazione affetta da HCV.

\begin{tabular}{|c|c|c|}
\hline DATABASE & CODICE & DESCRIZIONE \\
\hline \multirow[t]{11}{*}{ FARMACEUTICA } & J05AB04 & Ribavirina \\
\hline & L03ABIO & Interferone peghilato alfa- $2 b$ \\
\hline & L03ABII & Interferone peghilato alfa-2a \\
\hline & J05AEI2 & Boceprevir \\
\hline & J05AEII & Telaprevir \\
\hline & J05AXI5 & Sofosbuvir \\
\hline & J05AX65 & Sofosbuvir e Ledipasvir \\
\hline & J05AX67 & Ombitasvir, Paritaprevir, Ritonavir \\
\hline & J05AXI6 & Dasabuvir \\
\hline & J05AXI4 & Daclatasvir \\
\hline & J05AEI4 & Simeprevir \\
\hline SCHEDE DI DIMISSIONE & 070.41 & Epatite $\mathrm{C}$ acuta con coma epatico \\
\hline \multirow[t]{5}{*}{ OSPEDALIERA (SDO) } & 070.44 & Epatite $\mathrm{C}$ cronica con coma epatico \\
\hline & 070.51 & Epatite $C$ acuta senza menzione di coma epatico \\
\hline & 070.54 & Epatite $C$ cronica senza menzione di coma epatico \\
\hline & 070.70 & $\begin{array}{l}\text { Epatite virale } C \text { non specificata senza menzione di } \\
\text { coma epatico }\end{array}$ \\
\hline & 070.71 & Epatite virale $C$ non specificata con coma epatico \\
\hline ESENZIONE & 016 & Epatite virale \\
\hline
\end{tabular}

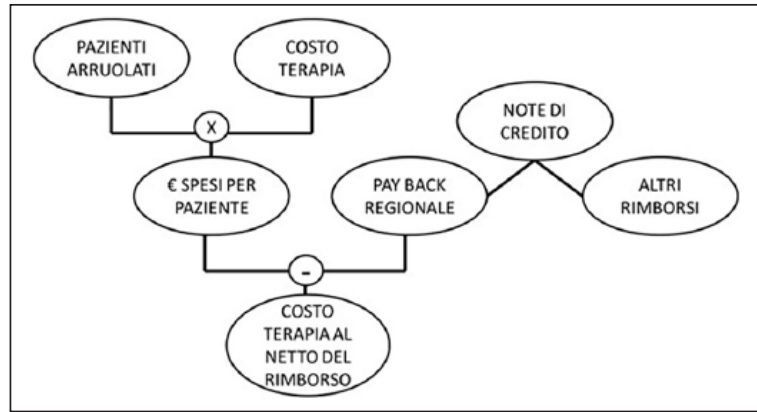

Figura I. Procedimento di calcolo per la valutazione dei costi delle terapie.

l'utilizzo di interferone associato a una indagine clinica correlata all'HCV.

Infine, per inquadrare la completa storia clinica del paziente si sono utilizzate le banche dati delle viste specialistiche, database contenente data e codice della visita e il costo; e il database degli esami ematochimici, contenente oltre al codice, al costo e alla data dell'esame anche l'esito. Grazie a questo è stato possibile individuare il valore della carica virale e quindi l'esito della terapia.

Si sono analizzate le cartelle cliniche di reparto (Unità Operativa Complessa di Malattie Infettive e Gastroenterologia) presenti in formato cartaceo per i soli pazienti seguiti dalle Malattie Infettive e Gastroenterologia del presidio ospedaliero dell'ULSS 2, al fine di migliorare e integrare le informazioni già riportate dal database amministrativo informatizzato, per esempio la data di diagnosi, genotipo ed esito del trattamento, non presenti nel data base amministrativo.

\section{Analisi dei dati}

Per l'analisi dei costi derivanti da prestazioni ospedaliere sono stati utilizzati i valori dei DRG e del costo delle visite specialistiche, riportate nel database.

Per l'analisi dei costi farmaceutici dei nuovi farmaci, non essendo a conoscenza delle scontistiche secretate inizialmente, si è resa necessaria la valutazione delle note di credito emesse dalle Aziende farmaceutiche. Le terapie innovative interferon-free, infatti, hanno determinato un elevato impatto economico nella fase iniziale della loro commercializzazione, e sono state soggette a meccanismi di pay-back da parte delle Aziende farmaceutiche sulla base del volume di farmaco ordinato, oltre che del numero di pazienti arruolati. Il calcolo è illustrato nella Figura 1.

Si è tentato di analizzare la gestione della patologia, nella sua complessità. Esaminando le cartelle cliniche dei pazienti con progressione di patologia, si è selezionato un campione della coorte che avesse almeno due diagnosi di stadi di fibrosi epatica consecutivi. Di questi è stato calcolato il periodo medio di permanenza in ciascun stadio e il costo medio sanitario ad esso associato.

Per questa analisi sono stati seguiti i pazienti dalla data di arruolamento allo studio, fino al 31/12/2016 o fino alla data di morte/trasferimento in altra azienda sanitaria. Considerando che la progressione della malattia è altamente eterogenea, si sono indicati i costi per ogni paziente, calcolando il valore medio annuo delle spese cliniche associate.

Per il calcolo delle visite specialistiche sono state considerate le visite inserite nell'esenzione $016,{ }^{16}$ mentre per la valutazione del costo delle complicazioni 
Tabella 2. DRG complicazioni epatiche.

\begin{tabular}{ll}
\hline DRG & INTERVENTO \\
\hline 480 & Trapianto epatico/intestino \\
I9I & Interventi su pancreas, fegato e di Shunt con complicanze \\
192 & Interventi su pancreas, fegato e di Shunt senza complicanze \\
205 & Malattie del fegato eccetto neoplasie maligne, cirrosi, epatite alcolica con complicanze \\
206 & Malattie del fegato eccetto neoplasie maligne, cirrosi, epatite alcolica senza complicanze \\
199 & Procedure diagnostiche epatobiliari per neoplasie maligne \\
200 & Procedure diagnostiche epatobiliari per neoplasie NON maligne \\
201 & Altri interventi epatobiliari o sul pancreas \\
\hline
\end{tabular}

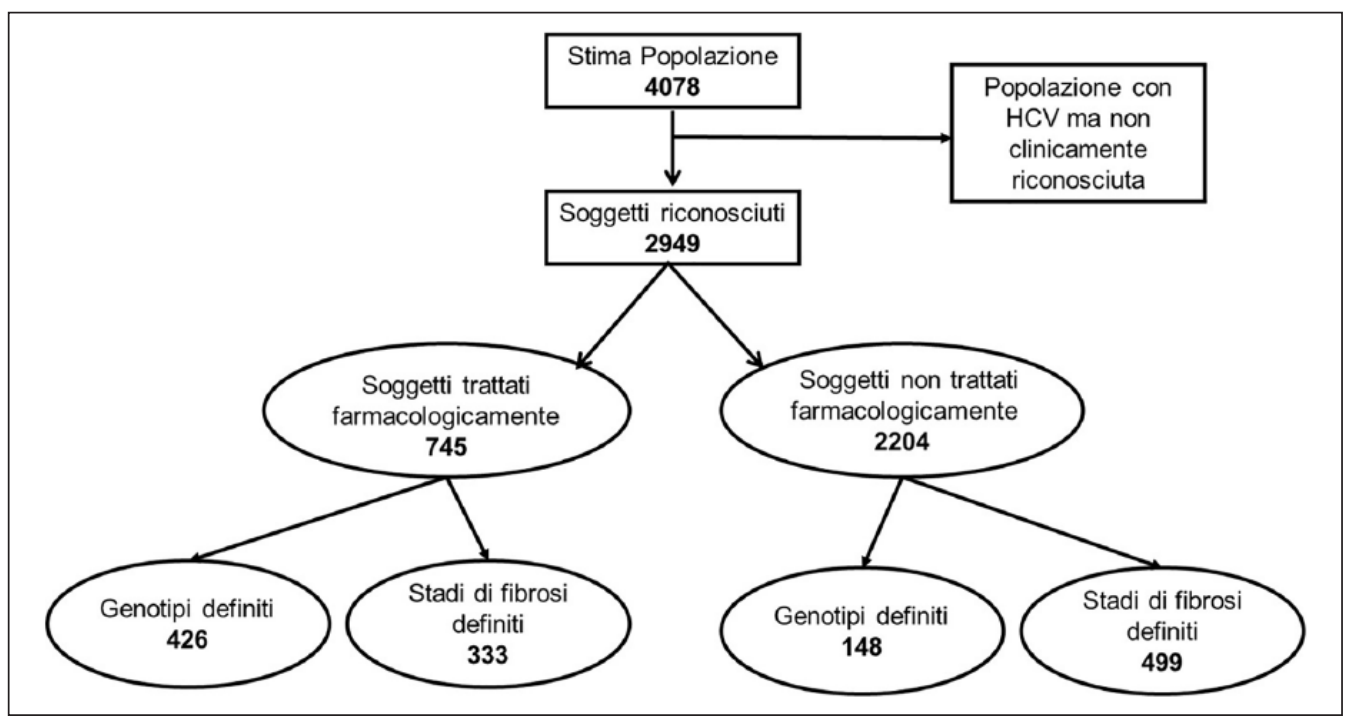

Figura 2. Diagramma di selezione della popolazione.

epatiche sono stati considerati i DRG presenti nella Tabella 2 .

Le analisi sono state eseguite con Microsoft ${ }^{\circledR}$ Excel $^{\circledR} \mathrm{e}$ Microsoft ${ }^{\circledR}$ Access $^{\circledR}$ per Windows ${ }^{\circledR}$ (Microsoft Corporation, Redmond, WA, USA).

\section{Risultati}

Nel corso dell'analisi, è emersa una prevalenza pari a 2.949 soggetti con almeno un accesso ospedaliero o trattamento farmacologico per cause correlate al virus, corrispondente al $63,57 \%$ di quanto inizialmente stimato da dati di letteratura. ${ }^{1,5}$ Considerando la prevalenza nei due sessi, si nota che nel 2015 negli uomini era dello 0,77\% mentre nel sesso femminile era dello $0,63 \%$.

La Figura 2 rappresenta schematicamente come è avvenuta la selezione del campione oggetto di studio: su 2.949 soggetti riconosciuti come portatori del virus $\mathrm{HCV}$, 745 sono stati trattati con farmaci, mentre per 2.204 non è stato tentato alcun approccio farmacologico.

Dalle cartelle cliniche di reparto è stato possibile caratterizzare una parte della popolazione sulla base del genotipo e del grado di fibrosi, ma non per tutti i pazienti erano presenti queste informazioni; per alcuni ne era indicata una ma ne mancava l'altra.

Come si evince dalla Figura 3, il genotipo 1 prevale rispetto agli altri. Complessivamente, nel corso degli anni dello studio (1997-2016) si presenta con una prevalenza del 57,75\%. Viceversa, il genotipo 4 è il meno rappresentativo nella popolazione prevalente $(8,74 \%)$, ma il più presente nella popolazione incidente.

Dall'analisi degli schemi terapeutici adottati nei soggetti affetti da epatite $\mathrm{C}$, emerge che circa un quarto della popolazione è stato trattato con almeno un farmaco antiepatite C (Figura 2), e di questi il 17,05\% dei pazienti ha eseguito più di un trattamento, fino a quattro tentativi di terapia).

Il totale di pazienti che sono stati trattati con terapie a base di interferoni è stato 653, di cui 50 con inibitori di proteasi e 277 con DAA. Questi ultimi erano vincolati dai criteri di eleggibilità AIFA, con lo scopo di modulare l'accesso ai pazienti con maggiore urgenza clinica al trattamento: il 94,09\% di essi, infatti, presentava una fibrosi medio grave (F3-F4). 


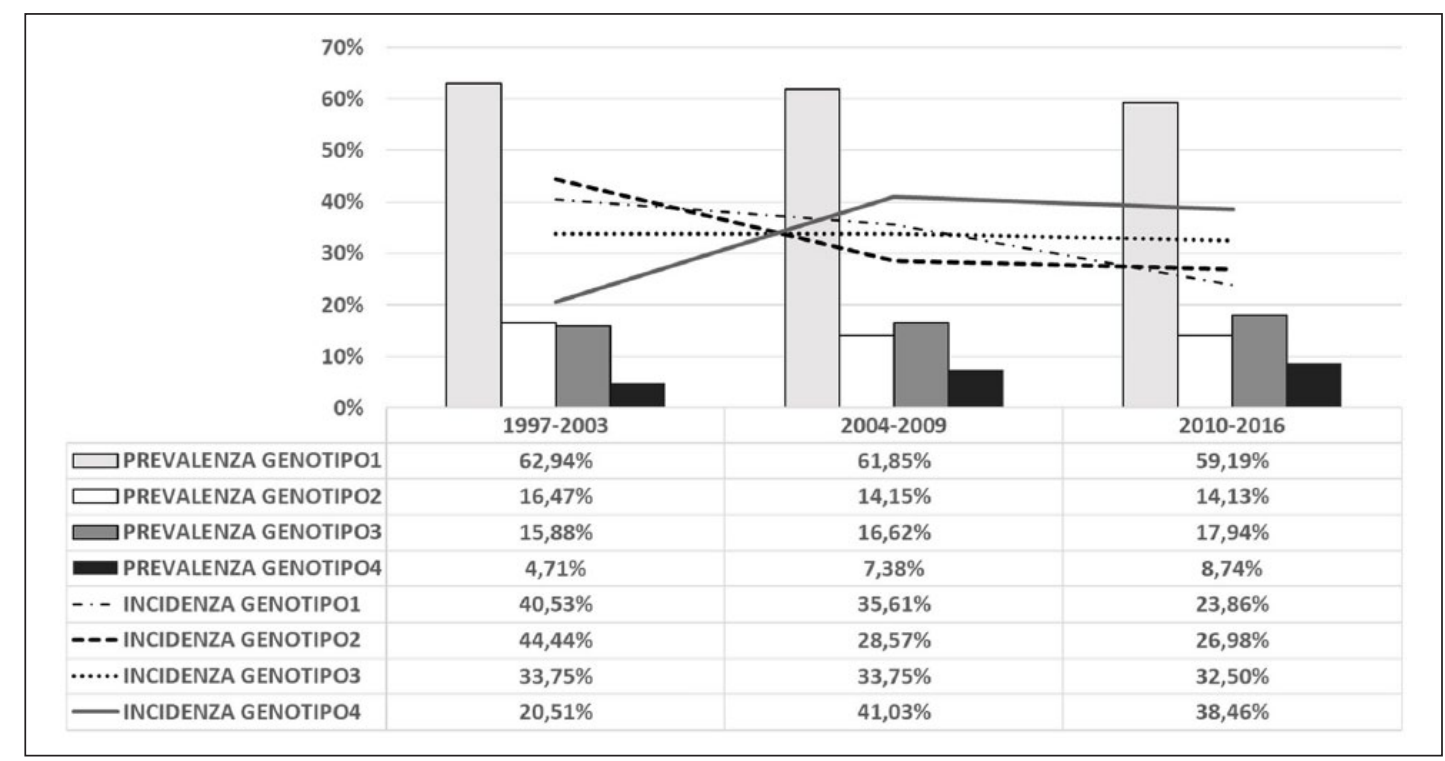

Figura 3. Incidenza e prevalenza dei genotipi dei pazienti afferenti all'ospedale di Treviso.

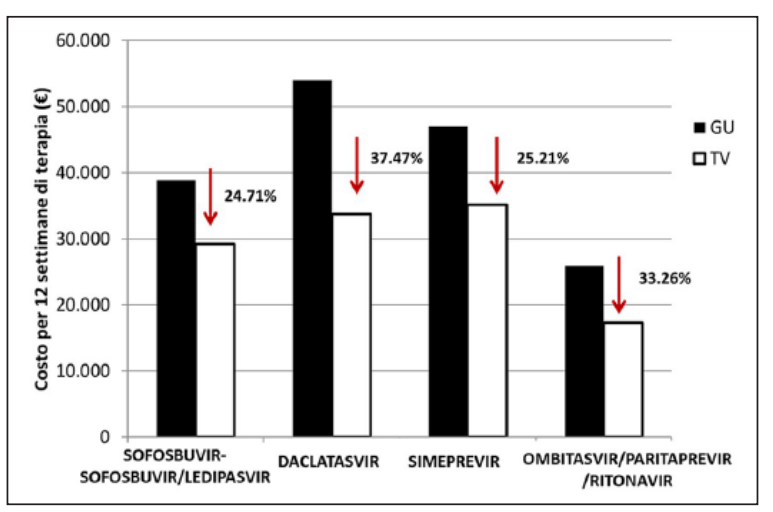

Figura 4. Risparmio medio sull'acquisto dei nuovi antivirali ad azione diretta (DAA).

Il raggiungimento della SVR si è ottenuto nel $27,75 \%$ dei soggetti trattati con interferone, nel $57,22 \%$ dei pazienti in triplice terapia e nel $98 \%$ dei soggetti trattati con i DAA.

Se il costo/terapia del regime a base di interferoni è di circa $€ 8.000 /$ ciclo terapeutico, il costo dei DAA è notevolmente superiore, soprattutto nella prima fase di commercializzazione; tuttavia gli accordi negoziali tra AIFA e le Aziende farmaceutiche hanno determinato una riduzione di spesa per l'acquisto dei farmaci DAA rispetto al costo degli stessi riportato in Gazzetta Ufficiale (GU).

Negli anni 2015-2016, l'Azienda ULSS 2 di Treviso ha registrato dei ritorni di spesa, grazie ai pay-back medi del $30 \%$ per ciclo di terapia considerando i nuovi regimi interferon-free: il grafico della Figura 4 riporta, per ciascun trattamento, la differenza di prezzo pubblicato in GU e quanto effettivamente sostenuto dall'Az. ULSS 2 di Treviso al netto degli sconti negoziali, derivanti dalle note di credito emesse dalle aziende.
L'arruolamento dei pazienti con i nuovi DAA è stato incrementale dal 2015 al primo semestre del 2016, per poi calare nel secondo semestre del 2016 (Figura 5).

Dall'analisi delle cartelle cliniche dei pazienti in trattamento con i DAA si è potuto evidenziare il genotipo di ciascun paziente e quindi è stato possibile stratificare il costo medio sulla base del genotipo (Figura 6). Considerando il primo periodo di utilizzo di questi nuovi farmaci (2015-2016) emerge che il genotipo 3 è il più costoso, quasi $€ 33.000 /$ ciclo di terapia, mentre $\mathrm{i}$ genotipi 1 e 4 superano di poco $€ 20.000 /$ ciclo di terapia, riducendo, in ogni caso, notevolmente il costo iniziale.

Infine, si è voluto analizzare la gestione della patologia, includendo i costi e la durata della patologia. Si sono considerati 823 pazienti su 2.949 , in quanto questa popolazione è ben caratterizzata sotto il profilo clinico, avendo almeno 2 individuazioni di stadi fibrotici differenti e ricerca della carica virale.

La stima media della durata dell'epatite $\mathrm{C}$ negli assistiti infetti dell'AULSS 2 di Treviso è di 26 anni, calcolata dalla sommatoria della durata media di ogni grado di fibrosi, per una spesa sanitaria globale superiore ai $€ 30.000$ (Figura 7). A questo importo si aggiungono i costi di terapia con DAA oppure, qualora non sia più possibile trattare il paziente, i costi di trapianto epatico, oltre al follow-up dopo la terapia. Il trattamento con DAA prevede un costo medio di $€ 24.000$, mentre il trapianto epatico viene a costare poco più di $€ 62.000$ (Decreto 18 ottobre 2012, Ministero della Salute e Ministero delle Finanze) (Figura 8).

Nella coorte della popolazione analizzata di questo studio, nell'arco dei 26 anni, si sono registrati 13 casi $(1,56 \%)$ di trapianto epatico correlati all'HCV. 


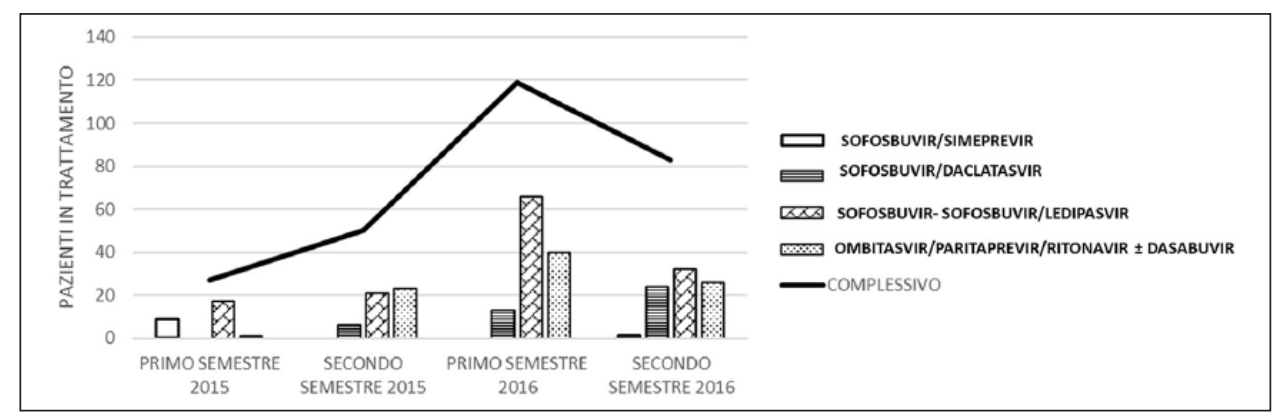

Figura 5. Arruolamenti agli antivirali ad azione diretta (DAA) avvenuti nel periodo dello studio.

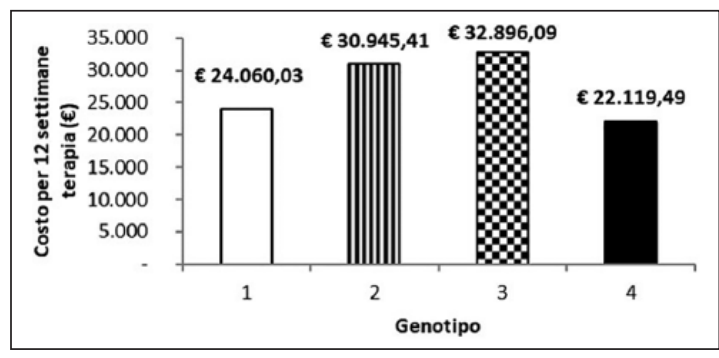

Figura 6. Costo per terapia per genotipo.

$\mathrm{Al}$ costo del trapianto sono stati associati anche i costi relativi al trattamento del tumore epatico. La chemioterapia in ospedale dei soggetti valutati e eventuali farmaci dispensati in distribuzione farmaceutica convenzionata raggiungono un valore medio di $€ 3.671$ per paziente trattato. Del presente studio il 29.68\% (247 soggetti) è progredito in epatocarcinoma.

Il costo di follow-up medio per un paziente trattato con DAA è di $€ 479 /$ anno circa, mentre la gestione di un paziente post-trapianto è di circa $€ 2.200 /$ annuo, inclusi i farmaci antirigetto e le visite specialistiche (Figura 8). Dallo studio delle banche dati emerge che l'aspettativa di vita di un paziente a seguito di trapianto epatico è di 7,6 anni, mentre risulta difficilmente calcolabile nei pazienti soggetti trattati con DAA data la recente commercializzazione.

\section{Discussione}

La prevalenza di pazienti affetti da epatite $\mathrm{C}$ residenti nell'Az. ULSS 2 di Treviso nell'anno 2015 è stata di poco superiore allo $0,50 \%$, differente dai dati di letteratura che riportano prevalenze maggiori all' $1 \% .{ }^{1,5}$

In riferimento ai dati di prevalenza emersi da altri studi, a poco più del $60 \%$ della popolazione affetta da $\mathrm{HCV}$ è stata diagnosticata la patologia. Tale percentuale è superiore alla stima globale elaborata dall'Osservatorio Polaris, il quale calcola che solo il $20 \%$ della popolazione affetta da epatite $\mathrm{C}$ ha avuto una diagnosi. ${ }^{17} \mathrm{E}$ noto che questa è una patologia molto lenta e silente ${ }^{18} \mathrm{e}$ in molti casi non dà manifestazioni cliniche fino a quando il paziente non è nello stadio di fibrosi avanzato.
Dal momento che per molti pazienti non è ancora chiara e definita la patologia, sarà importante poter identificare anche questa fascia di popolazione affetta, in quanto sono dei potenziali vettori del virus.

Fino a qualche anno fa la caratterizzazione del genotipo virale non era un'indagine di routine, a causa della poca selettività d'azione dell'interferone, unico trattamento farmacologico disponibile; la scelta terapeutica, quindi, non era effettuata in relazione al genotipo, come avviene attualmente, nonostante ci fossero genotipi più sensibili all'interferone stesso. ${ }^{19}$

La commercializzazione delle nuove terapie, invece, rende necessaria l'individuazione del genotipo nella scelta terapeutica più efficace. In linea con i dati epidemiologici nazionali ${ }^{5}$ anche nell'Az. ULSS 2 il genotipo 1 è prevalente (60\% circa), rispetto ad altri Paesi, come quelli africani, in cui domina il genotipo 4; è necessario monitorare eventuali variazioni genotipiche, anche in vista del flusso migratorio, che potrebbero determinare una lieve crescita dei genotipi 3 e $4 .{ }^{20}$ Un'altra motivazione della variazione di questi due genotipi è associata al fatto che sono veicolati principalmente tra coloro che fanno uso di sostanze d'abuso per via iniettiva, mentre il genotipo 1 è veicolato principalmente dalle trasfusioni ematiche non controllate. ${ }^{3}$

L'analisi dei trattamenti farmacologici iniziati dopo il 2000 nei pazienti residenti nell'Az. ULSS 2 di Treviso evidenzia che la maggioranza della popolazione non ha avuto alcuna prescrizione di farmaci correlati a epatopatie associate all'HCV. Alcuni autori hanno evidenziato che, in alcuni casi, i trattamenti farmacologici iniziano in stadio di progressione avanzato ${ }^{18,21,22}$ e che $\mathrm{i}$ pazienti che utilizzano sostanze psicoattive a uso iniettivo non trattati precocemente sviluppano complicazioni epatiche, incluso l'epatocarcinoma, in tarda età adulta. Ritardare il trattamento con i nuovi regimi farmacologici fino a stati di fibrosi avanzata prolunga il periodo di infettività.

Data l'asintomaticità della patologia, soprattutto nei primi stadi di fibrosi lieve-moderata, la diagnosi di HCV rappresenta spesso un riscontro occasionale che avviene solo in fase di malattia cronica. L'aumento della 


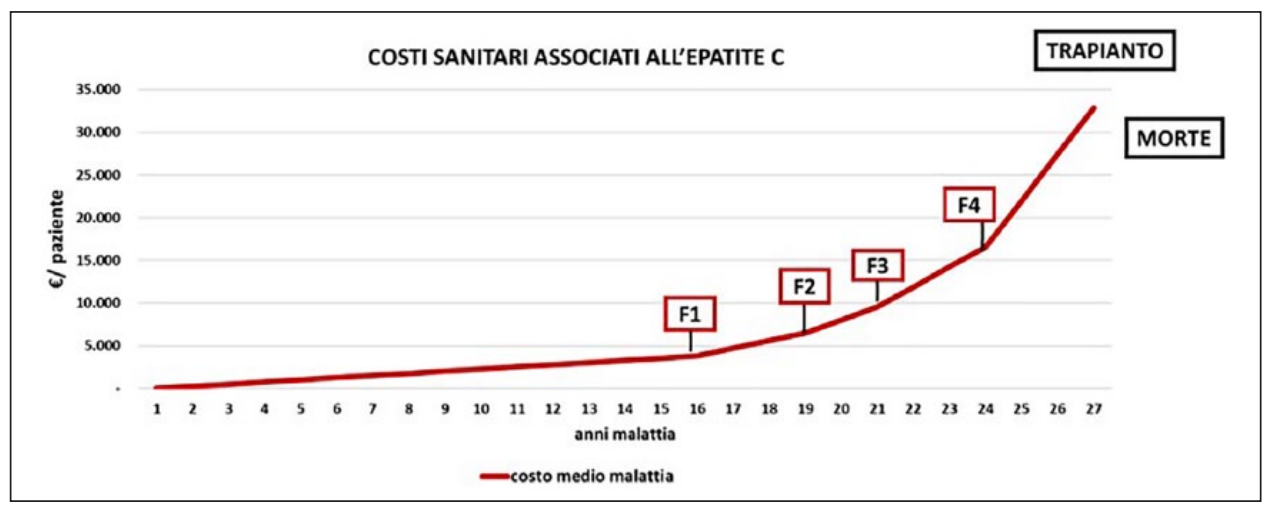

Figura 7. Costo sanitario cumulativo relativo alla malattia.

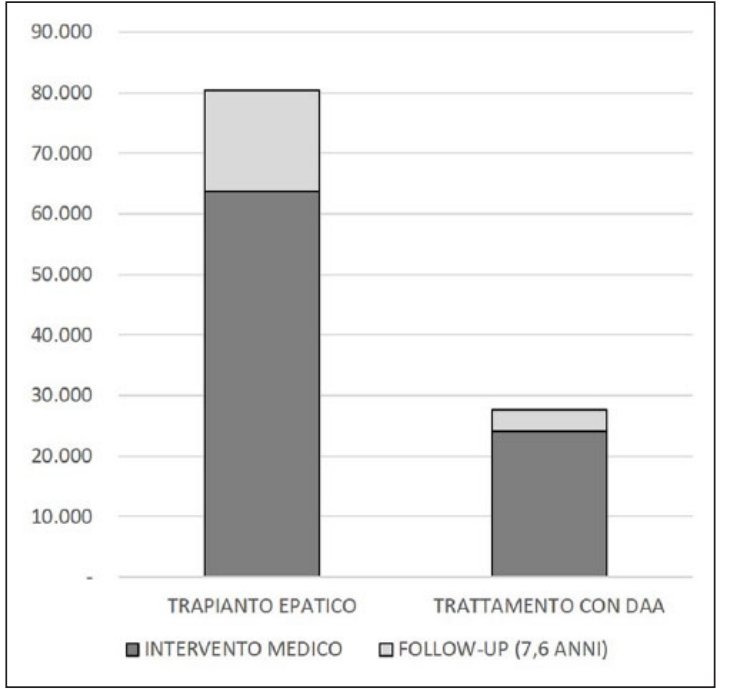

Figura 8. Confronto tra le spese associate al trapianto epatico e il trattamento con i farmaci antivirali ad azione diretta.

prevenzione e l'impegno precoce nella cura e nel trattamento potrebbero facilitare l'eliminazione del virus e la sua trasmissione, soprattutto nei pazienti tossicodipendenti. In passato, quando la sola terapia disponibile era l'interferone, molecola dai numerosi effetti avversi e dai tassi di guarigione relativamente bassi, ${ }^{21,23} \mathrm{i}$ pazienti dimostravano una notevole diffidenza al trattamento farmacologico.

Considerato il notevole impegno economico necessario per garantire le cure ai pazienti affetti da $\mathrm{HCV}$, si è reso necessario analizzare l'efficacia dei nuovi DAA, intesa come raggiungimento della SVR in condizioni di real practice: i farmaci erogati negli anni 2015-2016 hanno dimostrato un'efficacia migliore a quella espressa dai clinical trial, con performance superiori agli studi registrativi riportati nel riassunto caratteristiche del prodotto $(\mathrm{RCP}) .^{24}$

L'analisi economica dei costi globali della patologia è stata effettuata quantificando la durata media di ogni intervallo di fibrosi, dall'origine dell'infezione agli ultimi stadi della malattia. Limite di questa analisi è il criterio di scelta per la stadiazione della popolazione: non tutti i pazienti hanno avuto una registrazione della progressione della fibrosi, inoltre l'indagine clinica non è sempre puntuale e fornisce esiti corretti. ${ }^{25}$ Ancora, non è possibile quantificare l'intervallo temporale di transizione da uno stadio di fibrosi al successivo, a causa della eterogeneità di manifestazione del virus. ${ }^{26}$ Il modello utilizzato per la stadiazione della malattia può essere applicato per un'analisi di carattere economico, piuttosto che alla pratica clinica.

Sono stati considerati i costi sanitari diretti correlati alla progressione della patologia: un paziente costa al Servizio Sanitario Nazionale in media $€ 32.000$ nei 26 anni di infezione, escludendo il costo dei DAA e del trapianto di fegato che, fino a qualche anno fa, era considerato l'unico trattamento risolutivo. La durata della patologia è risultata inferiore rispetto allo studio di Massard che, tramite modello statistico, ha stimato una durata media di 30 anni. ${ }^{25}$ Un altro aspetto molto importante considerato nel presente studio è stato l'esito clinico della patologia nel paziente; in circa il $30 \%$ dei soggetti si è registrata l'evoluzione della malattia in carcinoma epatico, aggravante molto frequente nel paziente affetto da $\mathrm{HCV}$ e di cui sono stati valutati i costi.

Infine, si sono confrontati tra loro i costi di trapianto epatico e di trattamento con DAA, considerati i due unici interventi possibili per fronteggiare la malattia epatica causata dal virus dell'HCV in stadio cirrotico del fegato.

Nel primo caso è stato calcolato un costo da DRG di circa $€ 62.000$ per l'ospedalizzazione dovuta al trapianto, e i costi relativi a farmaci e visite specialistiche per i successivi 7 anni e mezzo; questi corrispondono all'aspettativa di vita dei pazienti trapiantati, analizzati nel presente studio.

Nel secondo caso sono stati valutati i costi relativi ai farmaci DAA, con un costo calcolato medio di $€ 24.000$ (dati del 2016). È da considerare che il costo unitario medio del trattamento per paziente ha subito una ulteriore 
netta riduzione nel 2017. Questo è dovuto al fatto che sono entrate in commercio nuove molecole, che hanno permesso di ridurre drasticamente i casi di relapser o non responder per tutti i genotipi riuscendo a raggiugere la SVR anche con una terapia dalla durata inferiore a 12 settimane. Per i pazienti trapiantati si sono valutati i costi successivi correlati al trattamento antivirale per un anno e quindi ricalcolati a 7 anni e mezzo per ottenere un confronto analogo tra $\mathrm{i}$ due gruppi. Emerge che il trattamento farmacologico è decisamente vantaggioso nel confronto con il trapianto dal punto di vista economico.

L'entrata in commercio dei DAA ha portato con sé molte polemiche dal punto di vista economico e morale. Questo perché il Servizio Sanitario Nazionale, assieme ad AIFA, aveva inizialmente limitato l'eleggibilità al trattamento ai soggetti più gravi (F3 e F4) a causa degli elevati costi iniziali. Avendo trattato molti pazienti nel primo semestre del 2016, si erano ormai ridotti i pazienti eleggibili; ciò potrebbe spiegare perché nell'ultimo semestre del 2016 si è registrato un calo del trattamento, in attesa dell'estensione dei criteri, avvenuta nel 2017.

Si è evidenziato, inoltre, un costo maggiore necessario per il trattamento dei genotipi G2 e G3 a causa di minori alternative terapeutiche, sofosbuvir e daclatasvir, rispetto agli altri genotipi (Figura 6).

Con l'estensione dei criteri di eleggibilità e con la graduale riduzione dei prezzi, avvenute durante la stesura del presente studio, è stato possibile trattare un maggior numero di soggetti affetti, e tale da poter procedere con l'avvio del piano di eradicazione regionale.

Si può sottolineare che il follow-up per i nuovi farmaci ad azione diretta sul virus, consiste in un controllo medico semestrale per 4 anni $^{27}$; le terapie con interferone, invece, prevedevano un controllo annuale per il monitoraggio della carica virale e il controllo della progressione della fibrosi epatica che si protraeva per tutta vita e, talvolta, senza raggiungimento della SVR.

Il presente studio mostra alcuni limiti. Tramite l'utilizzo delle banche dati non è possibile rintracciare tutte le informazioni per tutti i pazienti; per questo motivo si sono integrate alcune informazioni con l'utilizzo delle cartelle cliniche.

Non si conosce, poi, la reale data di incidenza della malattia, in quanto l'epatite $\mathrm{C}$ è una malattia silente e con conseguente difficoltà a diagnosticarla; inoltre, nel presente studio si è iniziato a rilevare i dati per singolo paziente, alla rilevazione di un evento indice (ricovero o prima terapia o rilevazione ematochimica), ma non al momento dell'infezione.

Non sono stati considerati $i$ costi indiretti della malattia ${ }^{28}$ in quanto non rilevabili tramite le banche dati, ed essendo questo uno studio retrospettivo osservazionale.

Sicuramente il dato presentato è sottostimato in quanto non tutta la popolazione è stata riconosciuta. Sarà interessante mettere a punto un algoritmo che permetta l'identificazione di questa popolazione al fine di porre in atto un piano di eradicazione della malattia.

Concludendo, i nuovi farmaci ad azione diretta, nonostante il costo iniziale elevato, permettono una risoluzione della malattia nella quasi totalità dei pazienti, per tutti i genotipi, con azzeramento della SVR. Il trattamento con DAA porta dei vantaggi sia per i pazienti che al Servizio Sanitario Nazionale.

Ora, l'obiettivo è di ricercare la quota delle persone affette ma che non ne sono a conoscenza, per poter eradicare completamente questa silenziosa ma fatale malattia.

\section{Declaration of Conflicting Interest}

The authors declare that there is no conflict of interest.

\section{Funding}

This research received no specific grant from any funding agency in the public, commercial, or not-for-profit sectors.

\section{Supplemental material}

Supplemental material for this article is available online.

\section{ORCID iD}

Annachiara Bellin (iD https://orcid.org/0000-0001-5494-6924

\section{References}

1. Gower E, Estes C, Blach S, et al. Global epidemiology and genotype distribution of the hepatitis $\mathrm{C}$ virus infection. $J$ Hepatol 2014; 61(1 Suppl): S45-S57.

2. Istituto Superiore di Sanità. Sistema Epidemiologico Integrato dell'Epatite Virale Acuta (SEIEVA). Andamento per anno delle incidenze [per 100000] di epatite C per età. http://old.iss.it/seieva (Ultima data di accesso al sito: 26/02/2019).

3. Esteban JI, Sauleda S and Quer J. The changing epidemiology of hepatitis C virus infection in Europe. J Hepatol 2008; 48(1): 148-62.

4. Wasley A and Alter MJ. Epidemiology of hepatitis C: geographic differences and temporal trends. Semin Liver Dis 2000; 20(1): 1-16.

5. Petruzziello A, Marigliano A, Loquercio G, et al. Hepatitis $\mathrm{C}$ virus (HCV) genotypes distribution: an epidemiological up-date in Europe. Infect Agent Cancer 2016; 11: 53.

6. Shire NJ and Sherman KE. Epidemiology of Hepatitis C Virus: A Battle on New Frontiers. Gastroenterol Clin North Am 2015; 44(4): 699-716.

7. Cacouba P, Gragnanie L, Comarmonda C, et al. Extrahepatic manifestations of chronic hepatitis C virus infection. Dig Liver Dis 2014; 46 Suppl 5: S165-S173.

8. Westbrook RH and Dusheiko G. Natural history of hepatitis C. J Hepatol 2014; 61(1): S58-S68.

9. Bjøro K, Bell H, Hellum KB, et al. Effect of combined interferon-alpha induction therapy and ribavirin on chronic hepatitis C virus infection: a randomized multicentre study. Scand J Gastroenterol 2002; 37(2): 226-232. 
10. Verbaan HP, Widell HE, Bondeson TL, et al. High sustained response rate in patients with histologically mild (low grade and stage) chronic hepatitis $\mathrm{C}$ infection. A randomized, double blind, placebo controlled trial of interferon alpha- $2 \mathrm{~b}$ with and without ribavirin, Eur $J$ Gastroenterol Hepatol 2002; 14(6): 627-633.

11. C. Cammà C, Petta S and Enea M. Cost-effectiveness of boceprevir or telaprevir for untreated patients with genotype 1 chronic hepatitis. Hepatology 2012; 56(3): 850-860.

12. Blasi R, Fantelli V, Andretta M, et al. Analisi della spesa e dei rimborsi relativi ai farmaci ad elevato impatto economico nella Regione del Veneto. Anno 2016. HTA Focus. Pills of Clinical Governance 2017; 4(2): 55-120.

13. Hill A, Khoo S, Fortunak J, et al. Minimum Costs for Producing Hepatitis C Direct-Acting Antivirals for Use in Large-Scale Treatment Access Programs in Developing Countries. Clin Infect Dis 2014; 58(7): 928-936.

14. Mennini FS, Marcellusi A, Viti R, et al. Disponibilità a pagare e innovazione: il caso dei farmaci anti-HCV nel Sistema Sanitario Italiano. GRHTA 2015; 2(2): 69-77.

15. European Association for the Study of the Liver (EASL). Clinical Practice Guidelines: Management of hepatitis C virus infection. J Hepatol 2011; 55(2): 245-264.

16. Unità di Informazione sul Farmaco. Libro Bianco, terza edizione, Verona, 2010.

17. Polaris Observatory, Polaris - Hep C - Dashboard, 2017. http://cdafound.org/polaris-hepC-dashboard/ (Ultima data di accesso al sito: 26/02/2019).

18. Moorman AC, Xing J, Ko S, et al. Late diagnosis of hepatitis $\mathrm{C}$ virus infection in the Chronic Hepatitis Cohort Study (CHeCS): Missed opportunities for intervention. Hepatology 2015; 61: 1479-1484.
19. Jacobson IM. Treatment options for patients with chronic hepatitis $\mathrm{C}$ not responding to initial antiviral therapy. Clin Gastroenterol Hepatol 2009; 7(9): 921-923.

20. Daw MA, El-Bouzedi A, Ahmed MO, et al. Epidemiology of hepatitis $\mathrm{C}$ virus and genotype distribution in immigrants crossing to Europe from North and sub-Saharan Africa. Travel Med Infect Dis 2016; 14(5): 517-526.

21. Vutien $\mathrm{P}$, Jin $\mathrm{M}$, Le $\mathrm{MH}$, et al. Regional differences in treatment rates for patients with chronic hepatitis $\mathrm{C}$ infection: Systematic review and meta-analysis. PLoS One 2017; 12(9): e0183851.

22. Sangiorgi D, Perrone V, Buda S, et al. Epidemiology, patient profile, and health care resource use for hepatitis C in Italy. Clinicoecon Outcomes Res 2017; 9: 609-616.

23. Jacobson IM, Poordad F, Brown Jr RS, et al. Standardization of terminology of virological response in the treatment of chronic hepatitis C: panel recommendations. J Viral Hepat 2012; 19(4): 236-243.

24. Yang YM and Choi EJ. Efficacy and safety outcomes of sofosbuvir-based treatment regimens for hepatitis $\mathrm{C}$ virusinfected patients with or without cirrhosis from phase III clinical trials. Ther Clin Risk Manag 2017; 13: 477-497.

25. Massard J, Ratziu V, Thabut D, et al. Natural history and predictors of disease severity in chronic hepatitis C. $J$ Hepatol 2006; 44(1 Suppl): S19-S24.

26. Deuffic-Burban S, Poynard T, et al. Quantification of fibrosis progression in patients with chronic hepatitis $\mathrm{C}$ using a Markov model. J Hepatol 2006; 44(1 Suppl): S114-S122.

27. EASL Recommendations on Treatment of Hepatitis C. European Association for the Study of the Liver (EASL) 2018; 69(2): 461-511.

28. Marcellusi A, Viti R, Capone A, et al. The economic burden of $\mathrm{HCV}$-induced diseases in Italy. A probabilistic cost of illness model. Eur Rev Med Pharmacol Sci 2015; 19(9): 1610-1620. 